\title{
Reconstruction the Postablative Lower Jaw Defects with Vas- cularized Free Fibula Flap with Further Endosteal Implants
}

\author{
Gagik Hakobyan, DMSc, $P h D^{1^{*}}$, Grigor Khachatryan, MD, PhD', Levon Khachatryan, MD, \\ PhD ${ }^{3}$ and Armen Hovhannisyan, MD, PhD ${ }^{4}$
}

${ }^{1}$ Head, Department of Oral and Maxillofacial Surgery, Yerevan State Medical University after M Heratsi, Armenia

${ }^{2}$ Associate Professor, Department of Oral and Maxillofacial Surgery, Yerevan State Medical University after M Heratsi, Armenia

${ }^{3}$ Head, Department of Maxillofacial and Plastic Surgery, Modern Implant Medicine, Armenia

${ }^{4}$ Head, Department of Plastic and Reconstructive Microsurgery, Erebouni Medical Center, Armenia

*Corresponding author: Gagik Hakobyan, DMSc, PhD, Head, Department of Oral and Maxillofacial Surgery, Yerevan State Medical University after M Heratsi, 0028 Kievyan str. 10 ap. 65 Yerevan, Armenia, Tel: +37410-271-146

\section{Abstract}

Objectives: To evaluate of the effectiveness of rehabilitation in patients following mandibulectomy and free fibula flap reconstruction with further endosteal implants. The patient cohort consisted of 27 patients years (14 males and 13 females) with lower jaw defects due to resection for tumors, were reconstructed with fibula-free flaps in a 6-year period (2015-2020). All patients underwent a thorough clinical laboratory, radiological examination according to a generally accepted scheme. Patients were evaluated by preoperative and postoperative outcome using computed tomography scan evaluation. The surgical procedure included: Segmentar resection of the lower jaw, reconstruction with fibula free flap. The implants were evaluated with measures of Resonance Frequency Analysis (RFA) during the follow-up periods using Osstell Mentor at time of implant placement, after 3 months. The mean RFA recordings of all 134 implants were 65 ISQ at implant placement respectively 73 ISQ after 3 months. After 4-6 months of healing, 152 dental implants were placed in non-irradiated fibular bone. Dental prosthetic rehabilitation was performed after 3-4 months of submerged healing. Patients had received implant-bridge and hybrid denture that provided ideal facial balance and occlusion. Postoperative clinical and radiographic controls were made regularly, the criteria for implant success were assessed. With dynamic observation, clinical and radiological indices were stable, of the 152 implants installed, 2 failed to osseointegrate and 5 after years of loading (peri-implantitis). Success rate of implants 5 years after was $96.1 \%$.

Conclusion: The reconstruction of the lower jaw defects after ablation of tumors with fibular flaps is a reliable method
\end{abstract}

with good long-term results. In all 27 patients, fibula flaps provided adequate bone stock for implant placement. Implants placed in the reconstructed areas were demonstrated to integrate normally. The results showed that implant treatment is effective to improve patients' masticatory efficiency and an acceptable quality of life to the patient.

\section{Keywords}

Free fibula flap, Reconstruction of the lower jaw, Endosteal implants, Prosthetic rehabilitation, Bone metabolism markers

\section{Introduction}

The mandibular resection resulting from treatment of malignant tumors, aggressive odontogenic tumors, or trauma can cause extensive composite defects including bone, oral lining mucosa, muscles, and teeth, with a significant decrease in the patient quality of life. After removal of the tumors the segmental bone defects initially cause a disruption of the muscular attachments, impair mastication and speech, and can lead to inadequate lip closure [1]. There are various methods available for reconstruction of mandibular defects: Non-vascularized bone grafts, titanium reconstructive splints or microsurgical techniques that allow the use of vascularized bone.

Every reconstructive technique must satisfy the fol-

Citation: Hakobyan G, Khachatryan G, Khachatryan L, Hovhannisyan A (2020) Reconstruction the Postablative Lower Jaw Defects with Vascularized Free Fibula Flap with Further Endosteal Implants. Res Rep Oral Maxillofac Surg 4:045. doi.org/10.23937/2643-3907/1710045

Accepted: November 07, 2020; Published: November 09, 2020

Copyright: (C) 2020 Hakobyan G, et al. This is an open-access article distributed under the terms of the Creative Commons Attribution License, which permits unrestricted use, distribution, and reproduction in any medium, provided the original author and source are credited. 
lowing key objectives: 1) Obliteration of the defect; 2) Restoration of function; particularly speech and mastication; 3) Structural support for reconstruction of external facial features; and 4) Aesthetic reconstruction of these external facial features [2]. The goals of mandibular reconstruction are to re-establish the form of the lower third of the face and to restore the patient's ability to eat in public, be intelligible to both trained and untrained listeners, and to maintain an unencumbered airway that allows the freedom to perform all activities [3]. Loss of mucosa from the floor of mouth is critical in the assessment of whether to restore this component of the defect with non-native tissue.

In cases of lesions of the lower jaw, segmental resection and reconstruction of the defect using a free flap of the fibula is a solution to restore the functions and aesthetics of the face resulting in a poor quality of life [4-6].

In 1989, Hidalgo became the first to report the transfer of fibular bone to reconstruct a segmental defect of the mandible [7].

Fibula is the most common donor site in both the vascularized and non-vascularized groups. Simple anatomy and accessibility of the fibula caused the popularity of this transplant in reconstructions of the lower jaw. The bone is available with enough length to reconstruct any mandible defect. A 22 to 25 centimeters segment of fibula bone may be harvested in the adult patient, permitting reconstruction of near-total mandibular defects with a single flap. During harvest, distally at least 5 $\mathrm{cm}$ of fibula should be left to prevent angle instability. It characteristics such as the thick and long cortical bony component (average width is $2.5 \mathrm{~cm} 3 \mathrm{~cm}$, thickness approximately $1.5 \mathrm{~cm}$ ) provide a good rigidity to withstand physiological stress during mastication. The peroneal artery and vein are usually of good quality and caliber and ideal for microsurgical anastomosis. Post ablative lower jaw defects with neoplasm resection can be performed in a single stage. It is considered the gold standard for the reconstruction of these types defect [8-10]. While surgical restoration of lower jaw has advanced dramatically with free-flap techniques, restoration of oral function requires advanced approaches. Restoration of oral functions requires not only reconstruction of the mandibular defect, but also dental rehabilitation [11-13].

In the field of fibula graft, dental implants have been widely used in recent decades [14-16]. Among the researchers, there is an unequivocal opinion about the timing of implantation. Some authors prefer the simultaneous installation of implants in the autograft, noting that the implants did not have a negative on the autotransplant remodeling process $[17,18]$.

In the case of a two-stage method, implants are placed 4-6 months after the autograft. According to many authors, the two-stage method is more predictable and reliable, because during this period, the regenerate occurs in the area of the autograft, which has good blood circulation, the autograft matures, converges in the recipient's area [19-23].

In the case of a larger defect, there is a risk of resorption due to the autograft to the implant or after orthopedic constructions [24].

Table 1: Patients gender, age, neoplasm type, reconstructed with fibula-free flaps.

\begin{tabular}{|c|c|c|c|c|c|c|c|}
\hline \multirow[b]{2}{*}{ Neoplasm Type } & \multirow[b]{2}{*}{$\mathrm{N}$} & \multirow{2}{*}{$\begin{array}{l}\text { Gender } \\
\mathrm{F} \\
\mathrm{M}\end{array}$} & \multicolumn{5}{|c|}{ Age of patients } \\
\hline & & & $21-30$ & $31-40$ & $41-50$ & $51-60$ & $61>$ \\
\hline Ameloblastoma & 8 & $\begin{array}{l}\text { F3 } \\
\text { M } 5\end{array}$ & 1 & 3 & 2 & 1 & 1 \\
\hline Osteoblastoclastoa & 7 & $\begin{array}{l}\text { F } 4 \\
\text { M } 3\end{array}$ & 0 & 2 & 3 & 2 & 0 \\
\hline Cementable fibroma & 3 & $\begin{array}{l}\text { F } 2 \\
\text { M } 1\end{array}$ & 1 & 2 & 0 & 0 & 0 \\
\hline Eosinophil granuloma & 2 & $\begin{array}{l}\text { F } 2 \\
\text { M } 0\end{array}$ & 0 & 1 & 1 & 0 & 0 \\
\hline Keratocyst & 2 & $\begin{array}{l}\mathrm{F} 0 \\
\mathrm{M} 2\end{array}$ & 1 & 1 & 0 & 0 & 0 \\
\hline Fibrous dysplasia & 2 & $\begin{array}{l}\text { F } 2 \\
\text { M } 1\end{array}$ & 0 & 1 & 1 & 0 & 0 \\
\hline Osteosarcoma & 3 & $\begin{array}{l}\text { F } 1 \\
\text { M } 2\end{array}$ & 0 & 0 & 0 & 1 & 0 \\
\hline Total & 27 & $\begin{array}{l}\text { F } 13 \\
\text { M } 14\end{array}$ & 5 & 10 & 7 & 4 & 1 \\
\hline
\end{tabular}


After transplantation of a vascularized fibular graft, the use of dental implants to restore chewing function is one of the solutions to this problem $[25,26]$.

Objectives to evaluate of the effectiveness of rehabilitation in patients following mandibulectomy and free fibula flap reconstruction with further endosteal implants.

\section{Material and Methods}

The patient cohort consisted of 27 patients years (14 males and 13 females) 26 and 63 presenting with lower jaw defects due to resection for tumors, were reconstructed with fibula-free flaps in a 6-year period (20152020).

The study was reviewed and approved by the Ethics Committee of the of the Yerevan State Medical University after M. Heratsi (protocol N12, 05.9.17) and in accordance with those of the World Medical Association and the Helsinki Declaration', this sentence could be a first sentence of the material-methods sections.

Clinical data included demographic and clinical variables: Gender; age; neoplasm type; location of the lower jaw defect (Table 1, Table 2 and Figure 1).

The Jewer DD l co-authorship classification model was used in the treatment of lower jaw bone defects [27].

Lower jaw defects according to Jewer DD 1) Central defect $C=$ central defect; 2 ) Bilateral defect $L C L=b i-$ lateral defect; 3) Central $\mathrm{L}$ lateral defect $\mathrm{LC}=$ central and lateral defect; 4) Hemimandibulectomy $\mathrm{H}=$ hemimandibulectomy and 5) Lateral defect $\mathrm{L}=$ lateral defect (Figure 1).

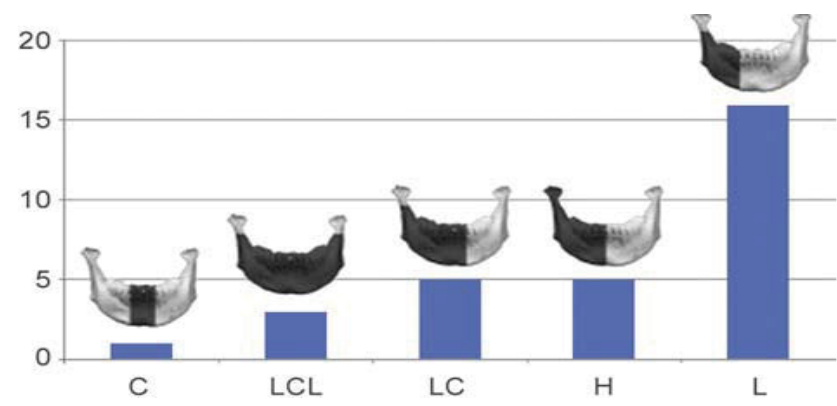

Figure 1: Lower jaw defects according to DD Jewer's classification model.
Patients with jaw lesions had different locations, the size of the lesion ranged from $2 \mathrm{~cm}$ to $23 \mathrm{~cm}$ (Table 3).

To assess the degree of osseointegration and determine the timing of the functional load of dental implants installed in free vascularized bone autografts, a Resonance-Frequency Analysis (RFA) of the stability of implants using Osstell Mentor (Osstell instrument, Integration Diagnostics AB, Gothenburg, Sweden) at time of implant placement, after 3 months. All patients presented functional and esthetic complaints. All patients underwent a thorough clinical laboratory, radiological examination according to a generally accepted scheme. Patients were evaluated by preoperative and postoperative outcome using computed tomography scan evaluation using CT Galileos (Sirona, German) (Figure 2, Figure 3, Figure 4, Figure 5 and Figure 6). Biopsy of the lesion was obtained in all cases. The choice of surgical treatment is determined by the type of lesions and the sizes of jaw defect.

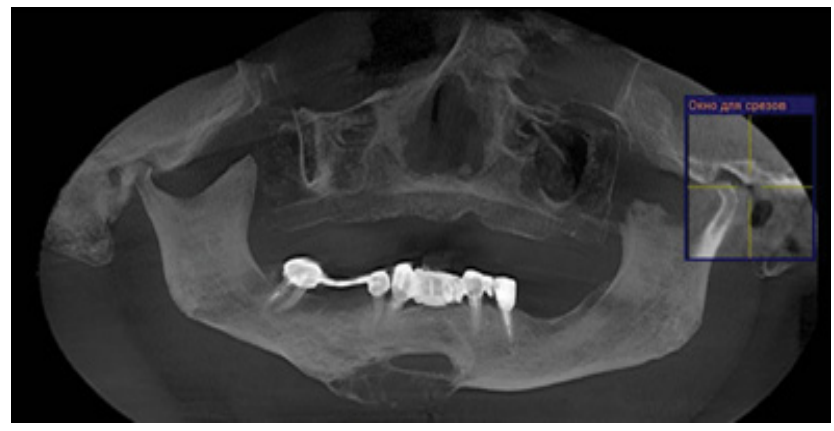

Figure 2: (Patients 1) Preoperative CT scan showing Low differential osteosarcoma (stage T4NO M0).

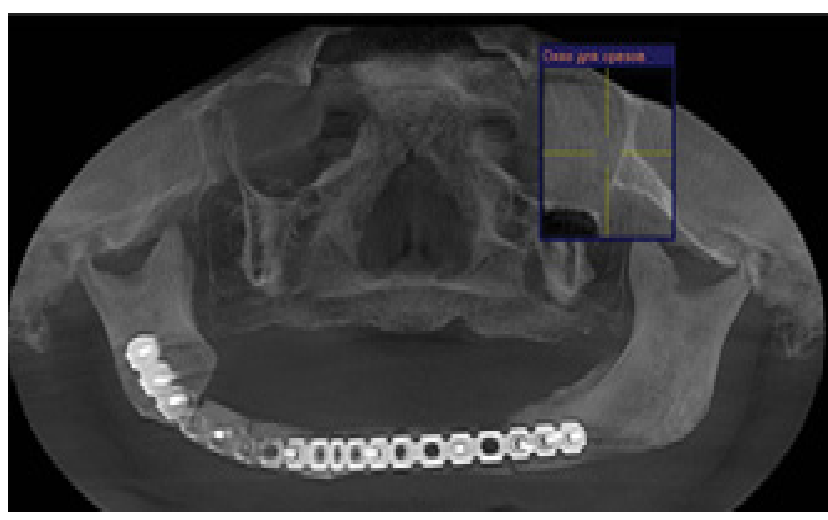

Figure 3: (Patients 1) CT scan after fibula transplant surgery.

Table 2: Distribution of patients according to the location of the lower jaw defect.

\begin{tabular}{|c|c|c|c|c|c|c|}
\hline \multirow{2}{*}{ Defect location } & \multirow{2}{*}{$n$} & \multicolumn{5}{|c|}{ Age of patients } \\
\hline & & $21-30$ & $31-40$ & $41-50$ & $51-60$ & $61>$ \\
\hline Central defect C & 3 & 0 & 1 & 2 & 1 & 0 \\
\hline Lateral defect L & 9 & 1 & 4 & 5 & 2 & 1 \\
\hline Bilateral defect LCL & 6 & 0 & 2 & 2 & 1 & 0 \\
\hline Central L lateral defect LC & 4 & 1 & 2 & 2 & 0 & 1 \\
\hline Hemimandibulectomy $\mathrm{H}$ & 5 & 0 & 0 & 1 & 1 & 1 \\
\hline
\end{tabular}




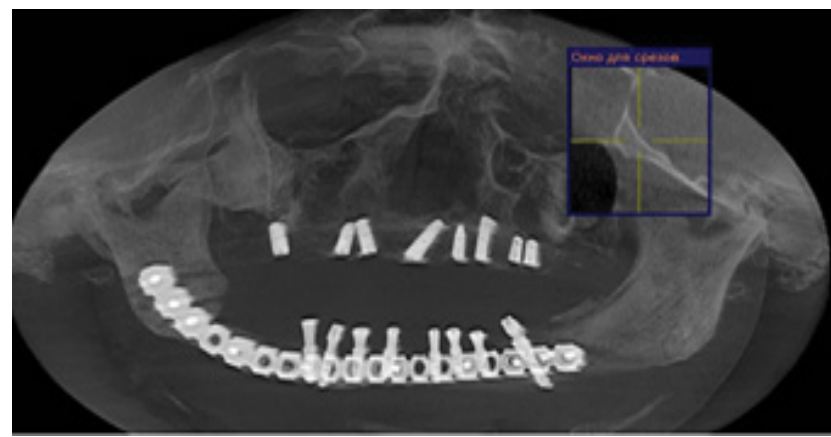

Figure 4: (Patients 1) CT scan showed well integrated dental implants in fibula transplant before prosthetic rehabilitation.

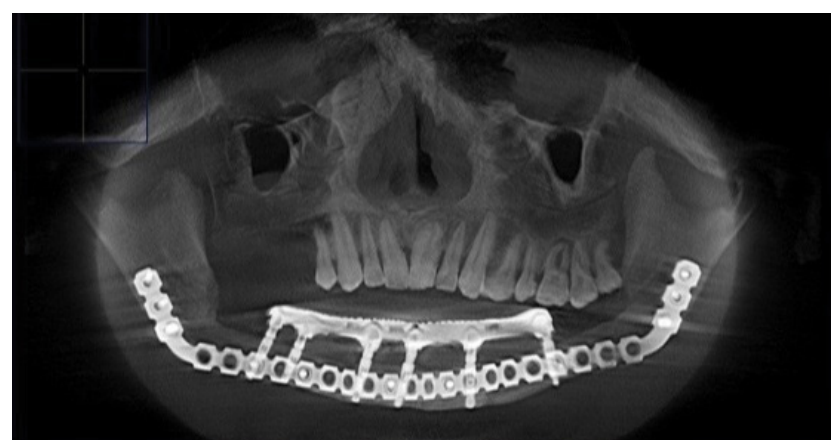

Figure 5: (patients 2) CT scan showed well integrated dental implants in fibula transplant after prosthetic rehabilitation.

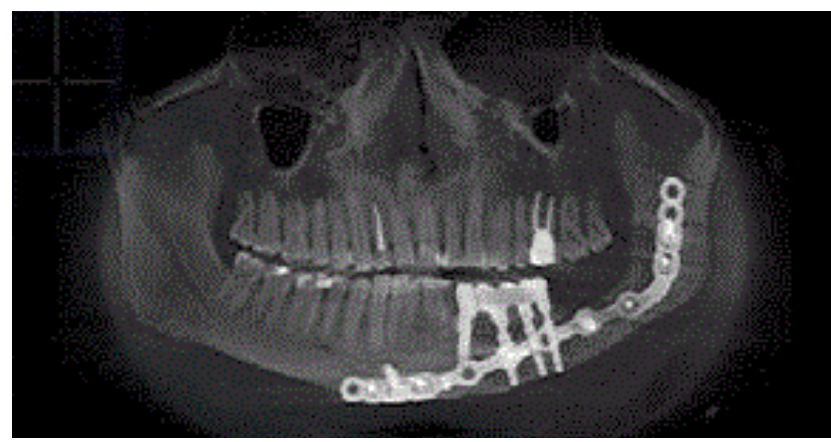

Figure 6: (Patients 3) CT scan showed well integrated dental implants in fibula transplant after prosthetic rehabilitation.

\section{Surgical Technique}

Two teams were involved in the surgery. The maxillofacial team resected the lesions of the lower jaw and further reconstruction with the fibular flap, the microsurgical team simultaneously harvested free vascularized flap of the fibula and performed an anastomosis of the arteries and veins of the fibula flap on the donor site. The surgical procedure included: Segmentar resection of the lower jaw, reconstruction with fibula free flap and implant installation 4-6 months after reconstruction (Figure 7, Figure 8, Figure 9 and Figure 10).

The protocol for functional reconstruction of mandibular defects consisted of several stages:

\section{Resection of the tumor,}

2. Reconstruction of the newly formed defect of the

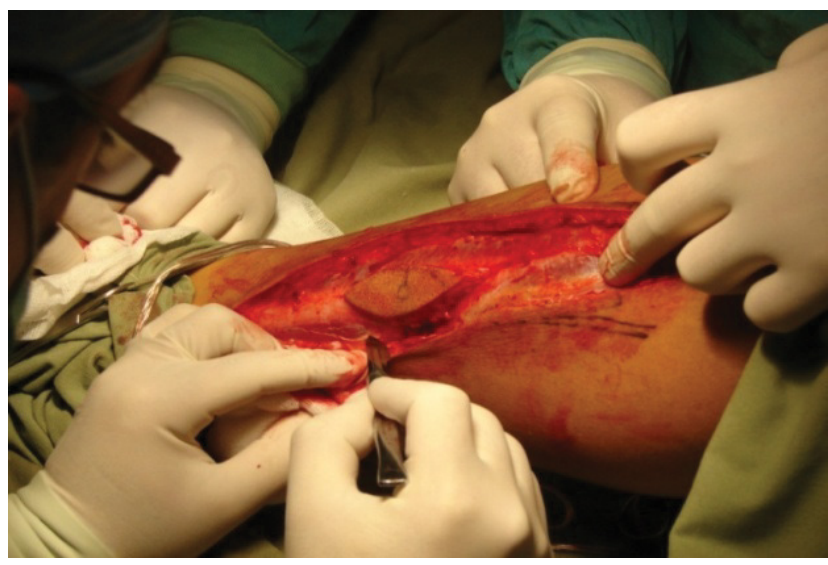

Figure 7: (Patients 1) Design skin island contour at harvest site of fibula bone.

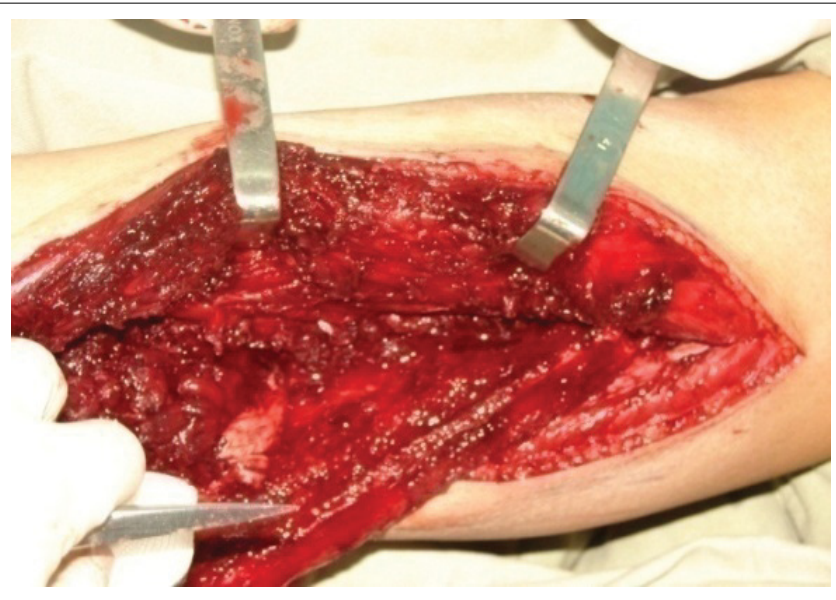

Figure 8: (Patients 1) Intraoperative photograph during harvesting the free fibula flap.

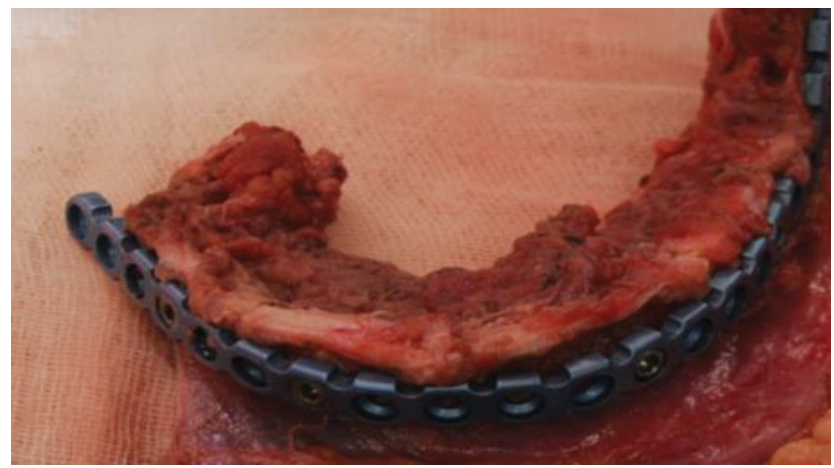

Figure 9: (Patients 1) The contoured fibula graft fixation with the titanium reconstruction plate.

lower jaw using a fibula flap with free edges together with defects of the lower jaw,

3. 6 months after resection of the mandible and reconstruction with a fibula flap installation of dental implants,

4. Above the installation of a denture 4 months after the installation of the implant.

The average length of the free fibula flap was 20$25 \mathrm{~cm}$, the thickness was $1.0 \mathrm{~cm}$, and the length of the vascular section was $10 \mathrm{~cm}$. The resulting free vascu- 


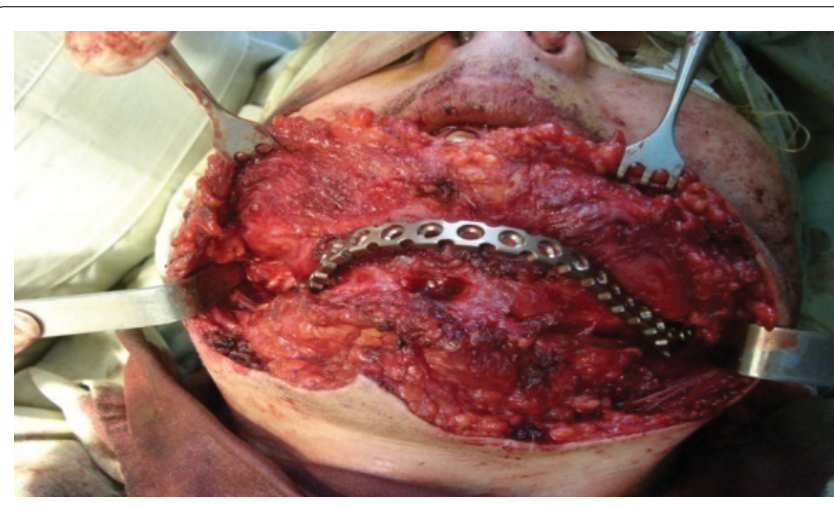

Figure 10: (Patients 1) Contoured fibula graft placed at recipient site and fixed to the mandibular bone by titanium plate and screws and imposition of the vascular anastomosis with $a / v$ facialis.
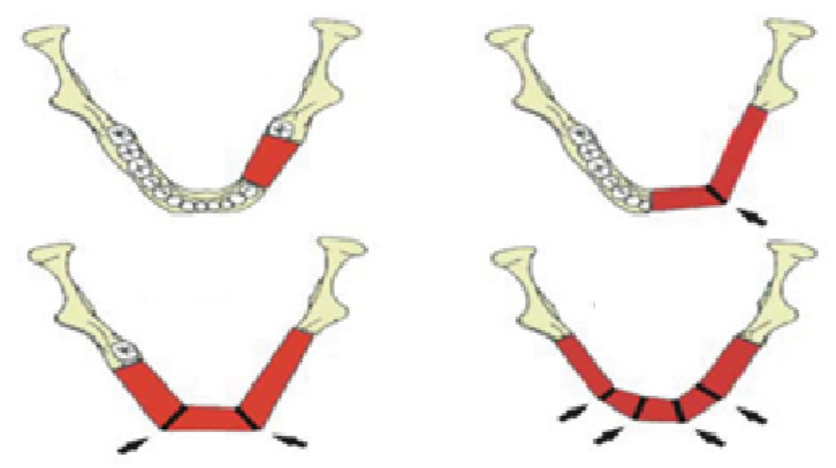

Figure 11: Free vascularized flap of the fibula segmentation scheme.

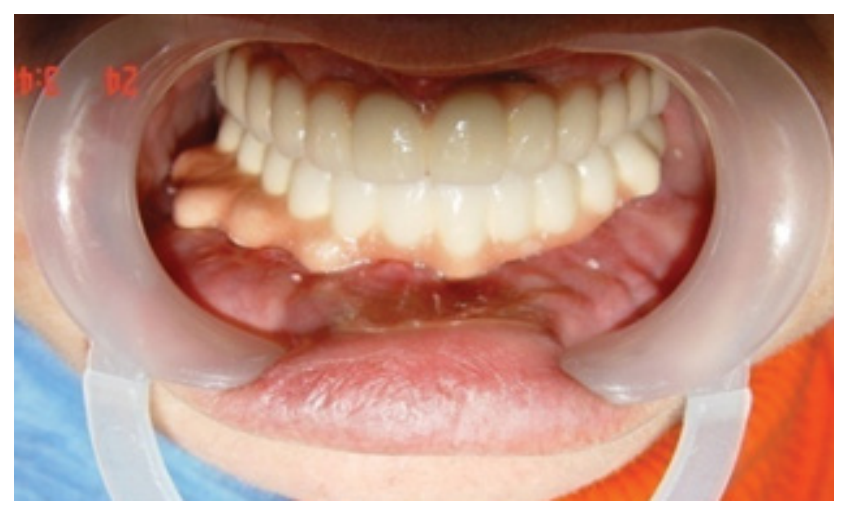

Figure 12: (Patients 1) Clinical appearance after prosthetic rehabilitation.

larized flap of the fibula was, if necessary, subjected to an osteotomy according to the size of the missing bone removed. The number of free fibula sections depended on the location of the bone defect $u$ was performed according to the scheme shown (Figure 11).

The simulated fibula autograft was fixed to the reconstructive bow with $1.2 \mathrm{~mm}$ diameter micro-screws.

The patient not received postoperative radiation therapy or chemotherapy. Postoperative clinical and radiographic controls were made regularly, using serial CT scan (Figure 3). After 4-6 months of healing, 152 dental implants were placed in the reconstructed mandible. Implants were uncovered 5-6 months after implantation. Before stage II surgery, a panoramic radiograph was taken to assess the stability of the bone level surrounding the implants and to ensure that there were no infections. Reconstructive and implant-prosthetic planning took using CT scan data and computer aided design (CAD) software.

Patients had received implant-bridge and hybrid denture that provided ideal facial balance and occlusion (Figure 5). The types of prostheses used varied among patients, depending on the need, occlusion and the number of implants that may be posted.

Successes of oral implant rehabilitation were: prosthesis success; implant success; complications; marginal bone loss (MBL).

Radiograph was used to detect any bony abnormality and evaluate alveolar bone around each implant and made as average value. Postsurgical change in marginal bone level was assess by digital $x$-ray were taken immediately (base line for comparison) and 3 months after implant installation, after prosthesis loading, 1 year, 3 years, and 5 years after implant installation (Figure 5 , Figure 6 and Figure 12).

\section{Statistical analysis}

Statistical analyses were performed using SPSS software ver. 22.0 (IBM, Armonk, NY, USA), and MedCalc program for Windows, $p$ values $<0.05$ were considered statistically significant.

\section{Results}

Clinical observations have shown that in some pa-

Table 3: Free fibula used according to the length of the defect.

\begin{tabular}{|l|l|l|}
\hline Defect length & N & $\begin{array}{l}\text { Free fibula used } \\
\text { according to the length }\end{array}$ \\
\hline Up to $5 \mathrm{~cm}$ & 7 & $25.95 \%$ \\
\hline $5-10 \mathrm{~cm}$ & 12 & $44.45 \%$ \\
\hline$>10 \mathrm{~cm}$ & 8 & $29.6 \%$ \\
\hline Total & 27 & $100 \%$ \\
\hline
\end{tabular}

Table 4: Early complication area after fibula transplantation.

\begin{tabular}{|l|l|l|}
\hline $\begin{array}{l}\text { Early complications of the } \\
\text { recipient period }\end{array}$ & $\mathbf{N}$ & $\mathbf{P} \pm \mathbf{~ m}$ \\
\hline Opening the edges of the wound & 0 & \\
\hline Bleeding & 0 & \\
\hline Pain & 20 & $33.9 \pm 6.1^{* *}$ \\
\hline Swelling & 20 & $33.9 \pm 6.1^{* *}$ \\
\hline Hematoma & 7 & $11.9 \pm 4.2^{*}$ \\
\hline Heat & 12 & $20.3 \pm 5.2^{* *}$ \\
\hline Total number of cases & 59 & 100 \\
\hline
\end{tabular}

${ }^{*} \mathrm{P}<0.05,{ }^{* *} \mathrm{P}<0.01$ 
tients included in the study, the surgical period passed without serious complications, and some patients developed various complications in the early postoperative period and later, as shown in Table 4 and Table 5. In the recipient area after fibula transplantation, all patients had swelling and pain in 7 patients, hematoma was observed.

The treatment objective was to achieve adequate esthetics profile, and to construct biomechanically favorable prosthesis to provide efficient masticatory function. No serious intraoperative or immediate postoperative complications were noted.

The first radiographic changes of the remnants of the remaining bone fragments and the transplant appear 50-70 days after transplantation and were completed 180 days after transplantation.

CT data on the state of the bone tissue after bone restoration surgery show that over time, bone tissue undergoes changes, gradually increasing in density over six months. In all 27 patients, fibula flaps provided adequate conditions for implant placement. Clinical investigation condition of the soft tissue the gingival, oral mucosa before implantation surgery is normal with sufficient thickness of the keratinized gum. The implants were osseointegrated, all the implants were stable and in good function with no post-operative complications.

After 6 months, the crestal bone loss of implants was evaluated by standardized periapical radiographs, the marginal bone loss (MBL) was $0.54 \mathrm{~mm}$ after 6 months, $0.97 \mathrm{~mm}$ after 12 months, after 36 months $1.23 \mathrm{~mm}$, after 60 months $1.43 \mathrm{~mm}$ (Table 6).

With dynamic observation, clinical and radiological indices were stable, of the 152 implants installed, 2 failed to osseointegrate and 5 after years of loading (peri-implantitis). Success rate of implants 5 years after was $96.1 \%$.

The analysis of the close-to-long-term results of implant-reconstructive operations performed in this work

Table 5: Delayed complications area after fibula transplantation.

\begin{tabular}{|l|l|}
\hline Delayed complications & $\mathrm{n}$ \\
\hline Transplant exposure & 0 \\
\hline Unscrewing the screws & 2 \\
\hline Transplant mobility & 0 \\
\hline Total number of cases & 2 \\
\hline
\end{tabular}

Table 6: The marginal bone loss (MBL) after fibula transplantation.

\begin{tabular}{|l|l|}
\hline Marginal bone loss (MBL) $\mathbf{n ~ ( 2 7 ) ~}$ & $\mathbf{m m}$ \\
\hline after 6 months & 0.54 \\
\hline after 12 months & 0.97 \\
\hline after 36 months & 1.23 \\
\hline after 60 months & 1.43 \\
\hline
\end{tabular}

suggests that a one-stage reconstructive operation, including dissection of the affected jaw, "simultaneous restoration of the defect with a vascular autograft", is a reliable method of reconstruction and makes it possible to orthopedic restoration of missing teeth using dental implants. The results of the study showed that there was a complete fusion of the two bone segments. The placement of an implant in the reconstructed bone site after reconstruction of the dissected part of the jawbone prevented unpredictable disintegration of the autograft made it possible to carry out orthopedic rehabilitation using implant-based structures.

\section{Discussion}

Current trends in mandibular reconstruction are aimed at restoring a viable mandible to the proper shape and the need for invasive autogenous graft production. Many reconstruction methods have been attempted and reported for the reconstruction of the mandible, which include reconstruction rods with or without pedunculated musculocutaneous flaps, alloplasts, and free grafts including partial or cortical bone, osteomyocutaneous pedicle flaps, and many free vascularized bone grafts. However, it should be noted that no method is ideal for the reconstruction of a mandibular defect $[1,28]$.

Optimal reconstruction of the mandibular defects remains controversial with regard to reconstructive options, which include donor site selection, timing of surgery, and recovery method [29].

The ideal timing for mandibular reconstruction is widely discussed, and today it is widely believed that immediate reconstruction can be performed without the risk of late diagnosis of disease recurrence [30].

Prior to the advent of microsurgical techniques, delayed reconstruction was critical for the maturation of the wound bed for nonvascular bone grafting [31,32].

Immediate reconstruction has advantages over delayed mandibular reconstruction. Health-related quality of life (QOL) studies have demonstrated that immediate reconstruction significantly improves quality of life and that the majority of patients prefer immediate reconstruction [33,34].

Boyd, et al. has shown in his work that patients who underwent reconstruction using vascularized bone grafts lost an average of 4 days of life due to secondary procedures, compared with 35 days for patients who underwent reconstruction with a soft tissue graft and flap [35].

The fibula can be used up to $25 \mathrm{~cm}$ of bone, and the bone supply is sufficient to support dental implantation. With this length of bone, the entire mandible can be reconstructed with vascularized bone if needed. Multiple osteotomies can be performed to reshape the fibula to restore the anterior arch, body, angle, or ramus of the mandible, provided the periosteum of the fibula is 
intact [1]. The skin on the lower side of the leg is thin and pliable, with quite a lot of available skin, and can be tolerated in a sensitive manner. The smaller skin blades can be used to close the defect first.

The leg of the free fibular flap is the peroneal artery, a branch of the tibial trunk of the fibula. The peroneal artery passes with paired comitant veins along the entire length of the fibula; along its medial aspect. The fibula feeds on both the periosteal and endosteal blood supply. It is this double blood supply that allows multiple osteotomies and contouring to be performed to repair large defects, including those at an angle. The vascular pedicle can be lengthened by taking the more distal segment of the bone, performing a subperiosteal dissection of the soft tissue surrounding the proximal bone, and ejecting it [36].

Technological advances in medical imaging and rapid prototyping make it possible to produce 3D models. 3D modeling of the bone graft also allows the creation of templates for contour osteotomy, which saves the surgeon time and maximizes bone-to-bone contact, promoting strong bone fusion. Mandibular reconstruction using computer-aided design and computer-aided manufacturing: an analysis of surgical results [37-40].

- The basic prerequisite for successful bone grafting [41].

- Bone transplantation into healthy tissue.

- Tight contact and fixation between the adjacent bone and the graft.

- Reception area with good blood supply.

Analysis of the long-term results of implantation-reconstructive operations performed in this work suggests that a one-stage reconstructive operation, including dissection of the affected jaw, "simultaneous restoration of the defect with a vascular autograft, is a reliable method of prevention, much lower." The number of traumatic operations hides the continuation of treatment and makes it possible to orthopedic restoration of missing teeth with dental implants.

Performed bone-restoration surgeries all patients had a positive result, fibula autograft adhesion to the residual jawbone was observed.

The bone segment obtained as a result of the use of fibula autogenous bone grafts with its quantitative-qualitative parameters was sufficient for the use of intraosseous implants.

Out of 196 implants, 7 implants were removed at different times ( 3 during osteointegration, 1 after surgery, 2 after 3 years, 1 after surgery) 96.5\%. The reason for the removal of the implants was periimplantitis, which was registered in patients who were left out of the dispensary control.

The results of the study showed that there was no disturbance in the nutrition of the remnant bone fragments of the small-vessel vascular transplant $u$ complete fusion of the two bone fragments. Implant placement in the reconstructed area of the bone after reconstructing the dissected part of the jaw prevented the unpredictable destruction of the autograft $u$ enabled orthopedic rehabilitation to be performed using implant-based structures.

\section{Conclusion}

The reconstruction of the lower jaw defects after ablation of tumors with fibular flaps is a reliable method with good long-term results. The results showed that implant treatment is effective to improve patients' masticatory efficiency and an acceptable quality of life to the patient. The multidisciplinary approach proved to be effective in overcoming the challenges.

\section{Conflict of Interest and Financial Disclosure}

The author declares that he has no conflict of interest and there was no external source of funding for the present study. None of the authors have any relevant financial relationship(s) with a commercial interest.

\section{Consent Statement}

Written informed consent was obtained from the patient for publication of this case report and accompanying images.

\section{Funding}

The work was not funded.

\section{References}

1. Batchu Pavan Kumar, V Venkatesh, KA Jeevan Kumar, B Yashwanth Yadav, S Ram Mohan (2016) Mandibular Reconstruction: Overview. J Maxillofac Oral Surg 15: 425-441.

2. Eric Santamaría, Peter G Cordeir (2006) Reconstruction of maxillectomy and midfacial defects with free tissue transfer. Journal of Surgical Oncology 94: 522-531.

3. Khachatryan G, Khachatryan L,Hakobyan (2018) The Treatment of Lower Jaw Defects Using Vascularized Fibula Graft and Dental Implants. The Journal of Craniofacial Surgery 29: 2214-2217.

4. Peled M, El-Naaj IA, Lipin Y, Ardekian L (2005) The use of free fibular flap for functional mandibular reconstruction. J Oral Maxillofac Surg 63: 220-224.

5. Mohammed Qaisi, James Murphy, Henry Fung (2019) Principles of Fibula Jaw in a Day Reconstruction: A Step-byStep Approach. Regenerative Strategies for Maxillary and Mandibular Reconstruction 211-233.

6. Runyan CM, Sharma V, Staffenberg DA, Levine JP, Brecht LE, et al. (2016) Jaw in a day: State of the art in maxillary reconstruction. J Craniofac Surg 27: 2101-2104.

7. Hidalgo DA (1989) Fibula free flap: a new method of mandible reconstruction. Plast Reconstr Surg 84: 71-78.

8. Kyung Chul Oh, a Ji-Hyun Park, Joon-hee Lee, Hong Seok Moon (2018) Treatment of a mandibular discontinuity defect by using a fibula free flap and an implant-supported fixed complete denture fabricated with a PEKK framework: A clinical report. J Prosthetic Dentistry 119: 1021-1024. 
9. Yetzer JG, Ettinger KS, Arce K, Salinas TJ (2017) Wholearch single-stage free flap reconstruction and rehabilitation of the mandible: a case report and technical considerations on a new technique. J Oral Maxillofac Surg 75: 290-296.

10. Salman SO, Fernandes RP, Rawal SR (2017) Immediate reconstruction and dental rehabilitation of segmental mandibular defects: description of a novel technique. J Oral Maxillofac Surg 75: 2270.

11. Papageorge M, SM Karabetou, LH Norris (1999) Rehabilitation of Patients with Reconstructed Mandibles Using Osseointegrated Implants. The Inter.Jof Oral Maxillofac Implants 14: 118-126.

12. Rutger H Schepers, Gerry M Raghoebar, Arjan Vissink, Martin W Stenekes, Joep Kraeima, et al. (2015) Accuracy of fibula reconstruction using patient-specific CAD/CAM reconstruction plates and dental implants: A new modality for functional reconstruction of mandibular defects. Journal of Cran Max Fac Surgery 30: 1-9.

13. Shantanu S, Mohit G Kheur, Satyajit Dandagi, Jay D Matani, Sumit Sethi (2015) Total Mandibular Reconstruction and Rehabilitation: A Case Report. Journal of Oral Implantology 12: 740-745.

14. Butura CC, Galindo DF, Jensen OT (2011) Mandibular allon-four therapy using angled implants: a three-year clinical study of 857 implants in 219 jaws. Oral Maxillofac Surg Clin North Am 23: 289-300.

15. Qaisi M, Kolodney H, Swedenburg G, Chandran R, Caloss $R$ (2016) Fibula jaw in a day: State of the art in maxillofacial reconstruction. J Oral Maxillofac Surg 74: 1284.

16. Almeida Moreira, Moreira MS, Farias BUL, Dias RB, Crossato EM, et al. (2015) Mandibular Reconstruction and Implantology: Anatomical Study and CT Scan of Dried Human Fibulas. Austin Journal of Dentistry 2: 1-4.

17. Jackson RS, Daniel L Price, Kevin Arce, Eric J Moore (2016) Evaluation of Clinical Outcomes of Osseointegrated Dental Implantation of Fibula Free Flaps for Mandibular Reconstruction. JAMA Facial Plastic Surgery 18: 201-206.

18. Anne-Gaelle B, Samuel S, Julie B, Lucas R, Breton $P$ (2011) Dental implant placement after mandibular reconstruction by microvascular free fibula flap: Current knowledge and remaining questions. Oral Oncol 47: 1099-1104.

19. João Ricardo Almeida Grossi, Tatiana Miranda Deliberador, Tielly Saad, Rafaela Scariot, Joao Cesar Zielak, et al. (2017) Oral and masticatory rehabilitation using osteointegrated dental implants after resective threatment of multicystic ameloblastoma in lower jaw with a fibula graft-a case report. Journal of Dentistry and Oral Disorders 3: 1-5.

20. Koudriavtsev V, Rodrigo A Jimenez (2018) Prosthetic challenges in a free fibula flap mandibular reconstruction: $A 10$ year follow-up of a case. Journal of Dental Implants 7: 64-69.

21. Silvio Mario Meloni, Marco Tallarico, Giacomo De Riu, Milena Pisano, Alessandro Deledda (2015) Guided implant surgery after free-flap reconstruction: Four-year results from a prospective clinical trial. Journal of Cranio-Maxillo-Facial Surgery 3: 1-8.

22. Bodard AG, Bémer J, Gourmet R, Lucas R, Coroller J, et al. (2011) Dental implants and free fibula flap: 23 patients. Rev Stomatol Chir Maxillofac 112: 1-4.

23. Johannes TM, Jan H Abbink, Robert $\mathrm{J} J$ van Es, Antoine J W P Rosenberg, Ron Koole, et al. (2018) Early and late complications in the reconstructed mandible with free fibula flaps. J Surg Oncol 117: 773-780.

24. Oteri G, Saverio De Ponte F, Pisano M, Cicciù M (2012) Five years follow-up of implant-prosthetic rehabilitation on a patient after mandibular ameloblastoma removal and ridge reconstruction by fibula graft and bone distraction. Dent
Res J (Isfahan). 9: 226-232

25. Wu YQ, Huang W, Zhang ZY, Zhang ZY, Zhang CP, et al. (2008) Clinical outcome of dental implants placed in fibula-free flaps for orofacial reconstruction. Chin Med J (Engl) 121: 1861-1865.

26. Jewer DD, Boyd JB, Manktelow RT, Zuker RM, Rosen IB, et al. (1989) Orofacial and mandibular reconstruction with the iliac crest free flap: a review of 60 cases and a new method of classification. Plast Reconstr Surg 84: 391-403.

27. Gullane PJ, Holmes H (1986) Mandibular reconstruction New concepts. Arch Otolaryngol Head Neck Surg 112: 714-719.

28. Urken ML (1991) Composite free flaps in oromandibular reconstruction. Review of the literature. Arch Otolaryngol Head Neck Surg 117: 724-732.

29. Schusterman MA, Harris SW, Raymond AK, Goepfert H (1993) Immediate free flap mandibular reconstruction: significance of adequate surgical margins. Head Neck 15: 204-233.

30. Maurer P, Eckert AW, Kriwalsky MS, Schubert J (2010) Scope and limitations of methods of mandibular reconstruction: A long-term follow-up. Br J Oral Maxillofac Surg 48: 100-104.

31. Lawson W, Loscalzo LJ, Baek SM, Biller HF, Krespi YP (1982) Experience with immediate and delayed mandibular reconstruction. Laryngoscope 92: 5-10.

32. Kramer FJ, DempfR, Bremer B (2005) Efficacy of dental implants placed into fibula-free flaps for orofacial reconstruction. Clin Oral Implants Res 16: 80-88.

33. Weymuller EA, Yueh B, Deleyiannis FW, Kuntz AL, Alsarraf R, et al. (2000) Quality of life in patients with head and neck cancer: lessons learned from 549 prospectively evaluated patients. Arch Otolaryngol Head Neck Surg 126: 329-335.

34. Vinzenz K, Schaudy C (2011) Osteoplastic surgery of the face-state of the art and future aspects. International Journal of Stomatology \& Occlusion Medicine 4: 142-156.

35. Boyd JB, Mulholland RS, Davidson J, Gullane PJ, Rotstein LE, et al. (1995) The free flap and plate in oromandibular reconstruction: long-term review and indications. Plast $\mathrm{Re}-$ constr Surg 95: 1018-1028.

36. Thoma A, Levis C, Young JE (2005) Oromandibular reconstruction after cancer resection. Clin Plast Surg 32: 361-375.

37. Rahimov CR, Farzaliyev IM, Fathi HR, Davudov MM, Aliyev A, et al. (2016) The application of virtual planning and navigation devices for mandible reconstruction and immediate dental implantation. Craniomaxillofac Trauma Reconstr 9: 125-133.

38. Okay DJ, Buchbinder D, Urken M, Jacobson A, Lazarus C, et al. (2013) Computer-assisted implant rehabilitation of maxillomandibular defects reconstructed with vascularized bone free flaps. JAMA Otolaryngol Head Neck Surg 139: 371-381.

39. Freudlsperger C, Bodem JP, Engel E, Hoffmann J (2014) Mandibular reconstruction with a prefabricated free vascularized fibula and implant-supported prosthesis based on fully three-dimensional virtual planning. J Craniofac Surg 25: $980-982$

40. Foley BD, Thayer WP, Honeybrook A, McKenna S, Press S (2013) J Oral Maxillofac Surg 71: 111-119.

41. Havlik RJ, MD Robert (1996) Reconstruction of the pediatric mandible. Oper Tech Plastic Reconstr Surg 3: 272-288. 